\title{
Neurosurgical applications of viscoelastic hemostatic assays
}

\author{
Svetlana Kvint, MD, ${ }^{1}$ James Schuster, MD, PhD, ${ }^{1}$ and Monisha A. Kumar, MD1,2 \\ Departments of ${ }^{1}$ Neurosurgery and ${ }^{2}$ Neurology, University of Pennsylvania, Philadelphia, Pennsylvania
}

Patients taking antithrombotic agents are very common in neurosurgical practice. The perioperative management of these patients can be extremely challenging especially as newer agents, with poorly defined laboratory monitoring and reversal strategies, become more prevalent. This is especially true with emergent cases in which rapid reversal of anticoagulation is required and the patient's exact medical history is not available. With an aging patient population and the associated increase in diseases such as atrial fibrillation, it is expected that the use of these agents will continue to rise in coming years. Furthermore, thromboembolic complications such as deep venous thrombosis, pulmonary embolism, and myocardial infarction are common complications of major surgery. These trends, in conjunction with a growing understanding of the hemostatic process and its contribution to the pathophysiology of disease, stress the importance of the complete evaluation of a patient's hemostatic profile in guiding management decisions. Viscoelastic hemostatic assays (VHAs), such as thromboelastography and rotational thromboelastometry, are global assessments of coagulation that account for the cellular and plasma components of coagulation. This FDA-approved technology has been available for decades and has been widely used in cardiac surgery and liver transplantation. Although VHAs were cumbersome in the past, advances in software and design have made them more accurate, reliable, and accessible to the neurosurgeon. VHAs have demonstrated utility in guiding intraoperative blood product transfusion, identifying coagulopathy in trauma, and managing postoperative thromboprophylaxis. The first half of this review aims to evaluate and assess VHAs, while the latter half seeks to appraise the evidence supporting their use in neurosurgical populations.

https://thejns.org/doi/abs/10.3171/2017.8.FOCUS17447

KEY WORDS viscoelastic; hemostatic; assays; coagulation; hemostasis; thromboelastography; rotational thromboelastometry

$\mathrm{V}$ ISCOELASTIC hemostatic assays (VHAs), such as thromboelastography (TEG) and rotational thromboelastometry (ROTEM), are global assessments of coagulation based on the physical and kinetic properties of clot formation. ${ }^{52}$ VHAs allow for rapid, point-of-care testing and identify all phases of hemostasis, from initial fibrin formation to clot lysis. VHAs provide a real-time analysis of hemostasis with information on the kinetics of clot formation and clot stability from the occurrence of the initial thrombin burst to fibrinolysis. Through measurement of the cellular, humoral, and fibrinolytic components of hemostasis, VHAs are able to identify both hypo- and hypercoagulable states. ${ }^{57}$

Traditional tests of coagulation, including the prothrombin time (PT) and partial thromboplastin time (PTT), reflect in vitro coagulation as assessed by the "cascade" model of coagulation..$^{42}$ Designed to evaluate clotting factor deficiencies, these tests reflect the time to initial thrombin generation and fail to reflect the subsequent clotting activity. ${ }^{8}$ The cell-based model accounts for the role of tissue-factor-bearing cells, platelets, and other cellular components in coagulation, which are omitted in the cascade

ABBREVIATIONS $\mathrm{AA}=$ arachidonic acid; $\mathrm{ADP}=$ adenosine diphosphate; $\mathrm{APTEM}=$ aprotinin effect as measured by $\mathrm{ROTEM}$; $\mathrm{CCT}=\mathrm{conventional}$ coagulation test; $\mathrm{CFT}=$ clot formation time; $\mathrm{Cl}=$ confidence interval; $\mathrm{DAT}=$ dual antiplatelet therapy; $\mathrm{DCl}=$ delayed cerebral ischemia; $\mathrm{DOAC}=$ direct oral anticoagulant; $\mathrm{EPL}=$ estimated percentage lysis; FF = functional fibrinogen; FFP = fresh frozen plasma; FIBTEM = fibrinogen component of ROTEM; HEPTEM = heparin effect as measured by ROTEM; ICH = intracerebral hemorrhage; INTEM = intrinsic pathway measured by ROTEM; LY30 = lysis at 30 minutes; MA = maximum amplitude; MCF = maximal clot firmness; ML = maximum lysis; $\mathrm{MRTG}=$ maximum rate of thrombus generation; $\mathrm{PED}=$ Pipeline embolization device; $\mathrm{PT}=$ prothrombin time; $\mathrm{PTT}=$ partial thromboplastin time; $\mathrm{RBC}=$ red blood cell; ROTEM = rotational thromboelastometry; RR = relative risk; SAH = subarachnoid hemorrhage; TBI = traumatic brain injury; TEG = thromboelastography; TMRTG = time to maximum rate of thrombus generation; TTG = total thrombus generation; TXA = tranexamic acid; VHA = viscoelastic hemostatic assay.

SUBMITTED July 1, 2017. ACCEPTED August 14, 2017.

INCLUDE WHEN CITING DOI: 10.3171/2017.8.FOCUS17447. 
model. VHAs are believed to better reflect the cell-based model of hemostasis, which may be more reflective of in vivo hemostasis, as compared with the traditional cascade model. ${ }^{19}$

Conventional coagulation tests (CCTs), including PT, PTT, D-dimer, fibrinogen, and platelet count, are often normal despite significant changes in coagulation function. ${ }^{48} \mathrm{CCTs}$ are performed using platelet-poor plasma and thus cannot provide information regarding clotting factor and platelet interactions, the final step in the creation of a stable clot. ${ }^{4}$ Understanding the contribution of platelets to hemostasis traditionally requires a variety of studies, including light transmission aggregometry, which are expensive and time consuming. ${ }^{23}$ The prolonged processing time associated with CCTs further limits their utility in the modification or individualization of patient therapy in emergent and perioperative settings. Despite these limitations, evaluation by CCTs has become the standard approach to assessing hemostasis and bleeding risk in the perioperative patient. ${ }^{23}$

In neurosurgery, it is critical to understand the individual patient's propensity for bleeding to preempt bleeding as well as to guide judicious resuscitation with transfusion of blood products. CCTs cannot adequately guide transfusion of blood components and thus may unnecessarily expose patients to adverse events..$^{20}$ It is clear that blood product transfusions are associated with significant morbidity and mortality.,17,49 Transfusion-related lung injury, transfusion-associated circulatory overload, hemolytic transfusion reactions, thromboembolism, and transfusiontransmitted infections are among the most serious of transfusion-related events. ${ }^{29,46}$ Not surprisingly, these complications contribute to prolonged patient hospitalizations and increased health care spending; in fact, transfusion-related adverse events in the US account for about $\$ 17$ billion in annual costs. ${ }^{56}$

\section{Thromboelastography and Rotational Thromboelastometry}

There are currently 2 leading FDA-approved VHAs: TEG 5000 [Haemoscope Corp.; FDA 510(k) no. K002177] and ROTEM [TEM International; FDA 510(k) no. K083842]. These assays use whole blood to assess the kinetics of clot evolution. Both assays determine a graphical output that represents clot formation and dissolution that is generated by the translation of torque along a torsion pin during thrombogenesis and fibrinolysis. ${ }^{52}$ The primary difference between the two concerns the movement of the cup, which holds the whole blood sample, and the sensor pin, which measures clot strength. Specifically, with TEG, the cup rotates while the pin is suspended freely in the cup, whereas with ROTEM these dynamics are reversed. ${ }^{52}$ Blood coagulation is initiated by the addition of an activating solution consisting of kaolin, phospholipids, and buffered stabilizers, which requires an activation phase before coagulation starts.

Although the mechanical principles of TEG and ROTEM are similar, the variability in hardware and reagents results in different output values and reference ranges; consequently, the generated results cannot be used interchangeably. ${ }^{57}$ Still, despite the discordant nomenclature, the parameters on the standard tracings are essentially the same (Table 1). ${ }^{53}$ Both modalities (Fig. 1) analyze clotting time, or the time until clot firmness reaches an amplitude of $2 \mathrm{~mm}$. This is prolonged by anticoagulants and shortened by hypercoagulable states. Both modalities measure clot kinetics, which is represented by the time it takes for the amplitude to grow from $2 \mathrm{~mm}$ to $20 \mathrm{~mm}$. This parameter is increased in hypofibrinogenemia. The $\alpha$ angle is homologous between the types of VHA and represents the slope between clotting time and clot kinetics. It is decreased with hypofibrinogenemia or platelet dysfunction. Clot strength is represented by the amplitude, and in the case of ROTEM is designated by a time duration (e.g., A10 is the amplitude at 10 minutes). Maximum clot strength is the greatest amplitude of the tracing, represented by the maximum amplitude (MA) or maximal clot firmness (MCF), and is decreased in platelet dysfunction or hypofibrinogenemia. Fibrinolysis is evaluated by clot lysis, both the lysis at 30 minutes (LY30) and estimated percentage lysis (EPL), which represent the percent decrease in amplitude 30 minutes after MA and estimated rate of change in amplitude after MA, respectively.

The ROTEM system also provides additional information about various aspects of the coagulation cascade. This includes EXTEM, which evaluates the extrinsic pathway akin to the prothrombin time; intrinsic pathway measured by ROTEM (INTEM), which evaluates the intrinsic pathway similar to the partial thromboplastin time; as well as fibrinogen component of ROTEM (FIBTEM), which evaluates the contribution of fibrinogen to clot formation. ${ }^{62}$ Assays that quantify the heparin and aprotinin effect (heparin effect as measured by ROTEM [HEPTEM] and aprotinin effect as measured by ROTEM [APTEM], respectively) are also available. These parameters, when used in combination, may indicate other derangements; for example, use of HEPTEM and INTEM may suggest heparin-induced coagulopathy. ${ }^{57}$

In addition to the standard analysis, the TEG system provides additional information about hemostasis. The difference in reaction time between a TEG performed in a heparinase cup versus that of a plain cup suggests a heparin-related anticoagulant effect. The functional fibrinogen (FF) parameter quantifies the contribution of fibrinogen on clot formation, somewhat similar to the FIBTEM. TEG offers both an estimate of platelet inhibition at the arachidonic acid (AA) and adenosine diphosphate (ADP) receptor sites and an estimate of thrombin generation. The estimate of platelet inhibition, termed "platelet mapping," assesses the patient's percentage platelet inhibition against his or her own maximum platelet function, as measured by changes in the MA due to antiplatelet therapy (e.g., acetylsalicylic acid, glycoprotein IIb/IIIa inhibitors, and clopidogrel). ${ }^{14}$ Thrombus velocity curves (V curve; Fig. 2.) represent the first derivative of the change in clot resistance and estimate the amount of thrombin generated with coagulation. ${ }^{53}$

There are potential advantages to VHA-guided evaluation of coagulopathy. Due to use of whole blood instead of plasma, actionable data may be available within 10-20 minutes as compared with 45-60 minutes for CCTs. Results may be generated within 5 minutes when using rapid 
TABLE 1. Comparison of TEG and ROTEM variables

\begin{tabular}{|c|c|c|c|}
\hline Measurement & $\begin{array}{c}\text { TEG } \\
\text { Parameter }\end{array}$ & $\begin{array}{c}\text { ROTEM } \\
\text { Parameter }\end{array}$ & Description \\
\hline \multicolumn{4}{|l|}{$\begin{array}{l}\text { Clot time: initiation to } \\
\text { 2-mm A }\end{array}$} \\
\hline & R time & CT & If abnormal, suggests deficiency of coagulation factors \\
\hline & SP & & Time from initiation to first detectable clot-related resistance \\
\hline & $\Delta$ & & Difference btwn the R time \& SP, a measure of hypo/hypercoagulability \\
\hline \multicolumn{4}{|l|}{$\begin{array}{l}\text { Clot kinetics: time from } \\
\text { 2- to } 20-\mathrm{mm} \mathrm{A}\end{array}$} \\
\hline & K & CFT & Measures enzymatic factors, anticoagulants, fibrinogen, platelets \\
\hline & $\alpha$ & $\alpha$ & Slope btwn R time \& K or CT \& CFT; measures fibrinogen, clot kinetics, platelets \\
\hline \multicolumn{4}{|l|}{ Clot strength (mm) } \\
\hline & A & A & A at standard times: 10,30 , or 60 mins (i.e., $\mathrm{A} 10, \mathrm{~A} 30$, or $\mathrm{A} 60$ ) \\
\hline & MA & MCF & $\begin{array}{l}\text { Measures maximal clot strength, assesses fibrinogen concentration, platelet count, platelet } \\
\text { function, factors VIII \& XIII }\end{array}$ \\
\hline & G & & Measure of total clot strength, calculated from MA \\
\hline \multicolumn{4}{|l|}{ Clot lysis } \\
\hline & LY30, EPL & CL30 & Measures fibrinolytic enzymes, fibrinolysis inhibitors, factor XIII at standard time (30 mins) \\
\hline & MRTG, TMRTG & & Estimate of thrombin generation \\
\hline Fibrinogen contribution & FF & FIBTEM & Qualitative assessment of fibrinogen levels \\
\hline Heparin effect & R time & HEPTEM & Detection of heparin; TEG = R time + heparinase cup, ROTEM = INTEM + heparinase \\
\hline Extrinsic pathway & & EXTEM & Measure of extrinsic pathway \\
\hline Intrinsic pathway & & INTEM & Measure of intrinsic pathway \\
\hline Aprotinin effect & & APTEM & Detection of fibrinolysis, done w/ EXTEM \\
\hline Ecarin effect & & ECATEM & Evaluation of direct thrombin inhibitors \\
\hline LMWH effect & R time & & Measures effect of LMWHs \\
\hline \multicolumn{4}{|l|}{ Antiplatelet effect } \\
\hline & MA-AA & & Platelet function (AA receptor) \\
\hline & MA-ADP & & Platelet function (ADP, glycoprotein IIb/IIla) \\
\hline
\end{tabular}

$\mathrm{A}=$ amplitude; $\mathrm{CL30}=$ clot lysis at 30 minutes; $\mathrm{CT}$ = clotting time; ECATEM = ecarin effect as measured by ROTEM; $\mathrm{K}=$ kinetics; LMWH = low-molecular-weight heparin; $\mathrm{R}$ time = reaction time; $\mathrm{SP}=$ split point; $\Delta=$ change.

TEG, in which tissue factor is used to expedite clotting. ${ }^{65}$ Evidence also supports the cost-effectiveness of VHAs compared with CCTs. ${ }^{66}$

Device-specific limitations may influence the preference of one VHA platform over the other. While the standard ROTEM device is automated and capable of analyzing 4 samples simultaneously, the TEG device is limited to 2 samples at a time. Additionally, the TEG requires manual pipetting, which can alter the results, and the machine is very sensitive to vibration. Additionally, the TEG 5000 system requires frequent quality controls. ROTEM and TEG are both device dependent; serial studies from the same patient should be run on the same machine..$^{25}$

An oft-cited limitation of VHAs concerns a perceived lack of validation. Robust correlations between VHAs and CCTs have been reported, such as between FIBTEM MCF and plasma fibrinogen level ${ }^{16}$ and between TEG MA and platelet count. ${ }^{2}$ However, because ROTEM and TEG tests use whole blood and not plasma, they are conducted differently from standard coagulation tests and thus they do not concur with plasma-based reference standards. For example, the FIBTEM assay and plasma fibrinogen concentration tests measure different physical properties, and furthermore, fibrinogen is not the only determinant of FIBTEM MCF. Simply put, they measure different aspects of hemostasis. Therefore, neither the US FDA nor the European Medicines Agency require that standard laboratory coagulation tests should be used as a reference method for ROTEM or TEG tests. ${ }^{59}$ ROTEM and TEG devices have been shown to meet the FDA standards of the comprehensive assessment of accuracy, precision, interference, reagent stability, and reference ranges, as well as software validation. ${ }^{59}$ Newer iterations of ROTEM and TEG, namely the ROTEM sigma and the TEG 6s, may be less encumbered by the perceived lack of validation or reliability. They are easier to use, have the potential to increase reproducibility, are less subject to operator error, and demonstrate improved quality control. ${ }^{59}$

\section{Utility of VHAs in Other Surgical Specialties}

Studies endorse the utility of VHAs to decrease blood product administration not only in the cardiac surgery population, but also in liver transplantation, trauma surgery, 


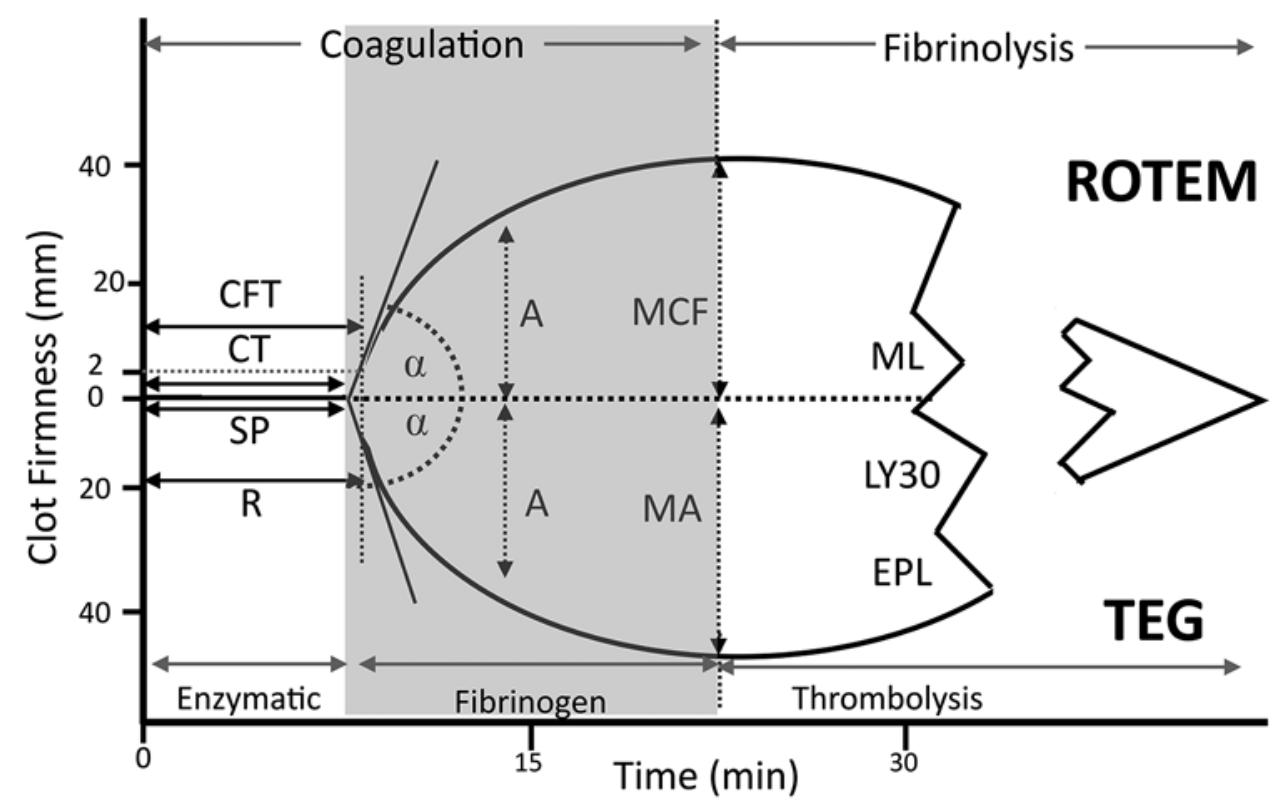

FIG. 1. Standard ROTEM (upper) and TEG (lower) VHA tracings. ROTEM value coagulation time (CT; in seconds) correlates with the TEG reaction time (minutes). These represent the contribution of clotting factors to clot formation or enzymatic reaction. ROTEM clot formation time (CFT; in seconds) correlates with the $\mathrm{K}$ value on TEG. The $\alpha$ angle is common to both and demonstrates the development of the fibrin and fibrin-platelet interactions. ROTEM maximum clot firmness (MCF, in $\mathrm{mm}$ ) correlates with the TEG maximum amplitude (MA; in $\mathrm{mm}$ ). ML on ROTEM is the percentage decrease in amplitude 60 minutes after MCF and is analogous to the LY30 and estimated percentage lysis (EPL) on TEG. Note that tracing is not to scale. $A=$ amplitude; SP = split point.

obstetrics, and orthopedics. ${ }^{1,67,68}$ A 2017 European metaanalysis that included 15 randomized controlled trials of more than 8000 cardiac surgery patients demonstrated that use of TEG or ROTEM resulted in the reduction of red blood cell (RBC) transfusion (relative risk [RR] 0.88,95\% confidence interval $[\mathrm{CI}] 0.79-0.97 ; \mathrm{I}^{2}=43 \%$ ) and platelet transfusion (RR $0.78,95 \%$ CI $0.66-0.93 ; \mathrm{I}^{2}=0 \%$ ). ${ }^{54} \mathrm{~A}$ 2016 Cochrane review of nearly 1500 patients, mostly in cardiac surgery, found that VHA-guided management was associated with a significant decrease in RBC transfusion (RR 0.86, 95\% CI 0.79-0.94; $\mathrm{I}^{2}=0 \%$ ), fresh frozen plasma (FFP; RR 0.57, 95\% CI 0.33-0.96; $\mathrm{I}^{2}=86 \%$ ), and platelet transfusion (RR $0.73,95 \%$ CI $0.60-0.88 ; \mathrm{I}^{2}=0 \%$ ). The review also found that TEG and ROTEM reduced overall mortality $\left(7.4 \%\right.$ vs $3.9 \%$; RR $0.52,95 \%$ CI $0.28-0.95, \mathrm{I}^{2}=$ $0 \%$ ), although only 8 studies provided data on mortality and the quality of evidence was deemed low. The authors concluded that application of TEG/ROTEM-guided transfusion strategies may reduce the need for blood product administration and improve morbidity in patients with bleeding but that this may be limited to patients undergoing cardiopulmonary bypass.

There has been an increase in the application of VHAs in trauma surgery. A meta-analysis of VHA-guided resuscitation in surgical patients $(n=1238)$ found that the volume of bleeding and the amount of transfused RBCs and FFP were significantly reduced in the VHA-guided group, although there was no difference for platelet transfusion requirements or mortality. ${ }^{9}$ Another review suggests that application of a VHA-guided transfusion strategy in patients with massive transfusion is associated with reduced bleeding but not improved morbidity or mortality. ${ }^{1,68}$ How- ever, a Cochrane review of VHAs in trauma concluded that TEG/ROTEM did not definitively diagnose early traumatic coagulopathy. ${ }^{21}$ Despite the conflicting data, the Trauma Quality Improvement Project of the American College of Surgeons suggests that all Level I and II trauma centers have TEG available..$^{51}$ Similarly, the European Trauma Guidelines suggest that ROTEM be performed to assist in the characterization of the coagulopathy and in guiding hemostatic therapy. ${ }^{50}$

\section{Utility of VHAs in Neurosurgery}

Given the potential utility of VHAs to identify the origin of bleeding and to guide blood component administration in other surgical specialties, it seems reasonable that there may be utility in the neurosurgical patient. The remainder of this review will explore the application and evaluate the extant literature of VHAs in neurosurgical populations.

\section{Vascular Neurosurgery \\ Intracerebral Hemorrhage}

Prompt identification of an anticoagulant effect is essential to the management of anticoagulant-associated intracerebral hemorrhage (ICH) as the duration of coagulopathy is associated with increased hemorrhage volume, and thus outcome. ${ }^{10}$ Early evaluation by VHAs may prove useful, as the effects of the direct oral anticoagulants (DOACs) are not readily assessed by CCTs. Specifically, while abnormal results on routine coagulation testing is consistent with the presence of a continued DOAC effect, normal values do not exclude clinically relevant concen- 


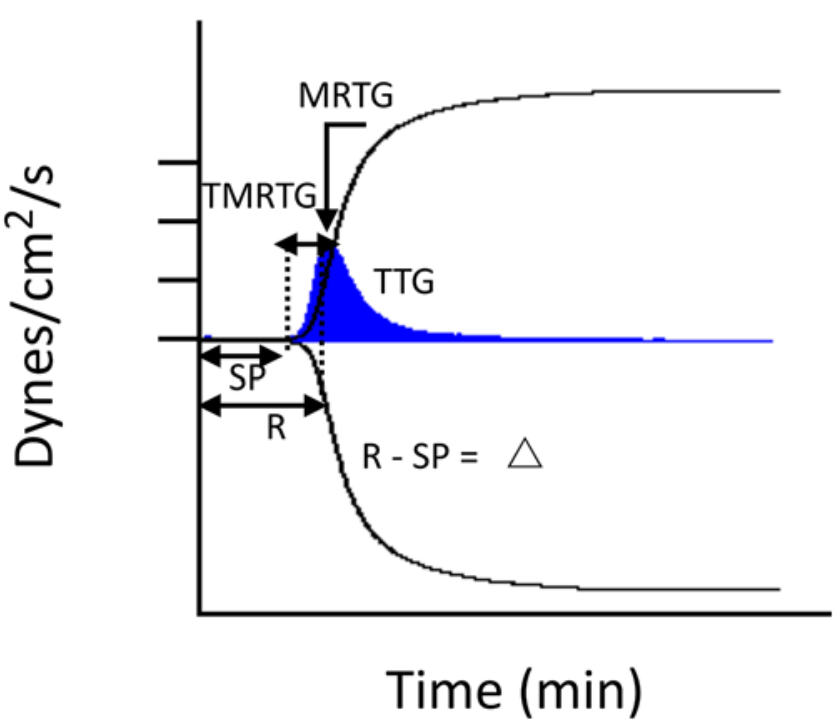

FIG. 2. TEG velocity curve. Sample of a thrombus velocity curve (V curve, in blue), calculated from the first derivative of changes in clot resistance, depicted here over a standard thromboelastographic tracing, showing the relationship of $\Delta=(\mathrm{R}-\mathrm{SP})$ and thrombus generation parameters. MRTG = maximum rate of thrombus generation; $R=$ reaction time; $\mathrm{SP}=$ split point; $\mathrm{TMRTG}=$ time to maximum rate of thrombus generation.

trations of these agents. ${ }^{13}$ There is some evidence to suggest the utility of VHAs among DOAC-treated patients, especially dabigatran. VHA parameters may correlate with plasma dabigatran concentration and effect, ${ }^{18,58}$ and rapid TEG may demonstrate a dose-response curve for dabigatran, rivaroxaban, and apixiban. Unfortunately, the activated clotting time of the rapid TEG, the only measure associated with the presence of the factor Xa inhibitors, is insensitive to low concentrations of these agents.?

VHAs may identify a subset of patients at risk for hematoma expansion. In a study of 64 patients with ICH and 57 controls, the ICH cohort was found to be more hypercoagulable than controls at baseline and 36 hours postictus. ${ }^{24}$ After controlling for potential confounders, admission $\mathrm{K}$ and $\Delta$ values were prolonged, indicating slower clot formation, in those patients who demonstrated hematoma expansion than those without. The authors suggest that this finding may indicate failure of an adaptive response, namely hypercoagulability, and may suggest a role for procoagulant strategies in patients at highest risk for expansion.

\section{Subarachnoid Hemorrhage}

Clinical management of subarachnoid hemorrhage (SAH) has centered on preventing and treating secondary injury after SAH, specifically delayed cerebral ischemia (DCI). DCI has been classically attributed to cerebral artery vasoconstriction in the setting of vessel irritation from local blood products. However, evidence suggests that DCI may result from a transient hypercoagulable state resulting from proinflammatory activation of coagulation. Ramchand et al. demonstrated a statistically significant increase in parameters of hypercoagulability, namely the MA $(p=0.032)$ and total thrombus generation (TTG; $p=$
0.013), in moderate to severe SAH versus healthy controls lasting for a period of 3-10 days after ictus. ${ }^{47}$

Evidence from VHA studies suggests that injury severity may affect platelet activation. In a study of more than 100 patients with SAH, mean MA was higher among patients with high Hunt and Hess grades (Grades 4 or 5) compared with Hunt and Hess Grades 1-3 within 72 hours of ictus $(\mathrm{p}=0.004)$. Furthermore, they were able to demonstrate a "dose-response" effect with increasing measures of platelet activation and inflammation associated with worsening Hunt and Hess grade. ${ }^{12}$

Other theories suggest that DCI results from early brain injury caused by pathophysiological events that occur at the time of aneurysm rupture. Studies performed early after SAH support the notion of a platelet-mediated hypercoagulable state by demonstrating a short $\mathrm{R}$ time and high MA when obtained within 30 minutes of SAH in animal models. ${ }^{28}$ Frontera et al. showed early ( $<72$-hour) elevations in MA to be associated with poor discharge disposition, longer length of stay, and worse functional outcomes in adult patients. ${ }^{12}$ As this relationship was not demonstrated with standard coagulation and inflammatory markers, MA is likely to be a more robust predictor of functional outcome than traditional markers of plateletmediated hypercoagulability. ${ }^{47}$

\section{Endovascular Neurosurgery}

The use of bare metal stents in endovascular treatment of aneurysms poses an immediate thromboembolic risk, necessitating dual antiplatelet therapy (DAT) before, during, and after the intervention until the thromboembolic risk normalizes. ${ }^{45}$ An optimal antiplatelet regimen allows for a delicate balance between the thromboembolic risk of a P2Y12 hyporesponse, and the hemorrhagic (and delayed thrombosis) risk of P2Y12 hyperresponse. However, adequate monitoring of antiplatelet activity is complicated by significant individual variability in platelet responsiveness to antiplatelet therapy. Yang et al. demonstrated that patients with a low MA-ADP were more likely to develop thromboembolism after treatment with a Pipeline embolization device (PED). ${ }^{71}$ McTaggart et al. used TEG platelet mapping to tailor a DAT induction strategy in patients treated with PED. ${ }^{36}$ Although further study is needed, these results suggest that TEG-guided DAT protocols may help to optimize the antiplatelet effect to improve outcomes among patients undergoing stent-assisted aneurysm treatment.

\section{Neurotrauma Surgery}

Traumatic brain injury (TBI) is associated with an occult coagulopathy. The reported incidence of occult coagulopathy may be as high as $87.5 \%{ }^{15,35,60}$ and is associated with worse clinical outcomes, such as increased mortality, higher likelihood of neurological decline at 24 hours, and higher rates of neurosurgical intervention. ${ }^{6,26,60}$ Windelov et al. demonstrated that hypocoagulable TEG values, defined by reaction time $>8$ minutes, angle $<55^{\circ}$, and/or MA $<51 \mathrm{~mm}$, correlated with poor prognosis and higher 30-day mortality in patients with isolated TBI. Interestingly, only 2 of 8 patients with hypocoagulability according to TEG had concurrent coagulopathy according 
to international normalized ratio, activated PTT, and/or platelet counts, which suggests that VHA values may be more reliable predictors of outcomes than CCTs. ${ }^{69}$ Davis et al. demonstrated increased platelet ADP and AA receptor inhibition among patients with TBI and showed that ADP inhibition distinguished survivors from nonsurvivors $(\mathrm{p}=$ 0.035 ), which correlated closely with severity of TBI ( $\mathrm{p}$ $=0.014){ }^{6}$ These data suggest that VHAs can be used to identify subgroups in the TBI population that would most benefit from earlier targeted therapies.

As premorbid use of anticoagulant or antiplatelet use is often unknown in the TBI population, VHA identification of antiplatelet and anticoagulant effect is a vital advantage. This is particularly important as antithrombotics influence the risk of traumatic hematoma expansion, and urgent surgical intervention, requiring assessment and treatment of coagulopathy, may be warranted..$^{32,40}$ Although individual rapid-TEG parameters failed to identify those patients at risk for hematoma expansion in a study of 279 patients with isolated TBI, the composite coagulopathy score, which was heavily weighted by rapid-TEG parameters, was associated with increased odds of expansion (OR 1.81, 95\% CI $1.09-3.01 ; \mathrm{p}=0.021) .{ }^{11}$ VHAs may improve outcomes by facilitating the prompt identification of hemostatic disturbances among patients with TBI treated with antithrombotic agents.

The association between TBI and a hypercoagulable state is also well established. ${ }^{43}$ Proposed etiologies include the protein $\mathrm{C}$ hypothesis, platelet hyperactivity, and extracellular vesicles. ${ }^{27}$ Massaro et al. demonstrated a progressive hypercoagulable state as evidenced by higher MA ( $p$ $=0.02)$, thrombus generation $(\mathrm{p}=0.03)$, and $\mathrm{G}(\mathrm{p}=0.02)$ values over the subsequent 5 days following initial TBI. ${ }^{34}$

Abnormalities in fibrinolysis may be common in trauma patients, but it is difficult to measure using routine CCTs. Nearly two-thirds of severely injured trauma patients demonstrate evidence of abnormal fibrinolysis by TEG..$^{38}$ Assessment of fibrinolysis is critical, because both suppressed fibrinolysis (termed "fibrinolysis shutdown") and hyperfibrinolysis increase mortality. ${ }^{5,38}$ Assessment with VHAs may guide antifibrinolytic therapy in patients with trauma and TBI.

\section{Tumor Neurosurgery}

Hypercoagulability is a common cause of morbidity and mortality among patients with intracranial tumors, and the use of VHAs in this population appears promising. ${ }^{54}$ The risk of venous thromboembolism ranges between $17 \%$ and $34 \%$ for high-grade astrocytic glioma during the postsurgical period, and the risk of VTE is similarly significant for other types of intracranial tumors. ${ }^{44,73}$ A study of 21 patients undergoing neurooncological surgery found that the ROTEM clot time could identify procoagulative activity of tumor tissue in vitro. ${ }^{22}$ Other potential applications of VHAs in neurosurgical oncology may be to guide postoperative thromboprophylaxis of brain tumor patients and aid in the reduction of VTE-associated morbidity and mortality.

VHAs may be useful to guide intraoperative blood transfusion in neurosurgical patients at risk for bleeding. Velez and Friedman reported a case of a patient with high- ly vascular meningioma who developed intraoperative disseminated intravascular coagulation, a rare but highly lethal complication. ${ }^{63}$ Intraoperative evaluation with TEG revealed significant fibrinolysis, which lead to treatment with aminocaproic acid. The patient fully recovered and is without focal neurological deficits. Similarly, Luostarinen et al. described two pediatric patients with large tumors who were evaluated by intraoperative ROTEM. ${ }^{33}$ They were considered high risk for intraoperative bleeding and empirically treated with FFP and RBCs. One developed an increased maximum lysis (ML) concerning for fibrinolysis requiring treatment with tranexamic acid (TXA), which would not have been evident with CCTs. The authors suggest that ROTEM offers a useful adjunctive tool to treat intraoperative coagulopathy in high-risk neurosurgical patients.

\section{Spine Neurosurgery}

The extent of perioperative blood loss associated with spine surgery may be significant and is determined by factors such as surgical time, primary versus repeat surgery, number of surgically treated levels, extent of soft-tissue exposure, single versus staged procedures, and whether an anterior and/or posterior approach is performed..$^{72}$ In a retrospective review of 423 patients undergoing 3-column resection osteotomies, Norton et al. reported major intraoperative blood loss (> $4 \mathrm{~L}$ ) in $24 \%$ of patients and an average percentage of total blood volume lost of $55 \% .{ }^{41}$

Fortunately, VHAs have demonstrated utility in the identification of volume expander solutions with a relatively low risk of hemostatic alterations. ${ }^{31}$ They have also allowed timely, factor-specific therapy through their rapid, realtime assessment of varying coagulation pathways. Mittermayr et al. reported improved ROTEM clot polymerization and strength with the use of fibrinogen concentrate in spine surgery patients with a dilutional coagulopathy, suggesting the importance of functional hypofibrinogenemia in major spine surgery. ${ }^{37}$ Relatedly, Naik et al. demonstrated that ROTEM-guided transfusion and early identification and management of hypofibrinogenemia were associated with reduced intraoperative blood loss, transfusion requirements, and transfusion-related cost. ${ }^{39}$ Furthermore, as prophylactic antifibrinolytic therapy has been shown to reduce perioperative blood loss in spinal surgery, VHAs may have a role in optimizing drug dosage and timing. ${ }^{30}$

\section{Pediatric Neurosurgery}

Major perioperative blood loss is a serious potential risk in pediatric neurosurgery. It often progresses to shock due to the patients' small blood volumes ${ }^{61}$ Although a number of transfusion strategies - preoperative erythropoietin treatment, prophylactic TXA, cell salvage, and prophylactic infusion of plasma-have been suggested to address the challenge of resuscitation in pediatric neurosurgery, none is without limitation. ${ }^{64}$ Volume preloading, for example, may increase the risk of intracranial hypertension or cerebral edema. Crystalloids and colloids may themselves interfere with coagulation and clot strength. Fortunately, while large trial data are lacking, some studies suggest improved intraoperative hemostasis using a VHA-guided strategy among pediatric neurosurgery patients. ${ }^{33,70}$ 


\section{Conclusions}

TEG and ROTEM, point-of-care assays of the viscoelastic properties of blood, provide a comprehensive realtime analysis of hemostasis, from initial thrombin burst to fibrinolysis, permitting improved transfusion strategies and resulting in the potential for goal-directed therapy of coagulation abnormalities in the perioperative neurosurgical period. This review describes the coagulation abnormalities associated with neurosurgical populations, underscores the limitations of the traditional approach to assessment of coagulopathy, and highlights the emerging role of VHAs in addressing these limitations.

\section{References}

1. Afshari A, Wikkelsø A, Brok J, Møller AM, Wetterslev J: Thrombelastography (TEG) or thromboelastometry (ROTEM) to monitor haemotherapy versus usual care in patients with massive transfusion. Cochrane Database Syst $\operatorname{Rev}(3): C D 007871,2011$

2. Alexander DC, Butt WW, Best JD, Donath SM, Monagle PT, Shekerdemian LS: Correlation of thromboelastography with standard tests of anticoagulation in paediatric patients receiving extracorporeal life support. Thromb Res 125:387-392, 2010

3. Baharoglu MI, Cordonnier C, Al-Shahi Salman R, de Gans K, Koopman MM, Brand A, et al: Platelet transfusion versus standard care after acute stroke due to spontaneous cerebral haemorrhage associated with antiplatelet therapy $(\mathrm{PATCH})$ : a randomised, open-label, phase 3 trial. Lancet 387:26052613, 2016

4. Bolliger D, Seeberger MD, Tanaka KA: Principles and practice of thromboelastography in clinical coagulation management and transfusion practice. Transfus Med Rev 26:1-13, 2012

5. Cotton BA, Harvin JA, Kostousouv V, Minei KM, Radwan $\mathrm{ZA}$, Schöchl H, et al: Hyperfibrinolysis at admission is an uncommon but highly lethal event associated with shock and prehospital fluid administration. J Trauma Acute Care Surg 73:365-370, 2012

6. Davis PK, Musunuru H, Walsh M, Cassady R, Yount R, Losiniecki A, et al: Platelet dysfunction is an early marker for traumatic brain injury-induced coagulopathy. Neurocrit Care 18:201-208, 2013

7. Dias JD, Norem K, Doorneweerd DD, Thurer RL, Popovsky MA, Omert LA: Use of thromboelastography (TEG) for detection of new oral anticoagulants. Arch Pathol Lab Med 139:665-673, 2015

8. Dzik WH: Predicting hemorrhage using preoperative coagulation screening assays. Curr Hematol Rep 3:324-330, 2004

9. Fahrendorff M, Oliveri RS, Johansson PI: The use of viscoelastic haemostatic assays in goal-directing treatment with allogeneic blood products - a systematic review and metaanalysis. Scand J Trauma Resusc Emerg Med 25:39, 2017

10. Flibotte JJ, Hagan N, O’Donnell J, Greenberg SM, Rosand J: Warfarin, hematoma expansion, and outcome of intracerebral hemorrhage. Neurology 63:1059-1064, 2004

11. Folkerson LE, Sloan D, Cotton BA, Holcomb JB, Tomasek JS, Wade CE: Predicting progressive hemorrhagic injury from isolated traumatic brain injury and coagulation. Surgery 158:655-661, 2015

12. Frontera JA, Provencio JJ, Sehba FA, McIntyre TM, Nowacki AS, Gordon E, et al: The role of platelet activation and inflammation in early brain injury following subarachnoid hemorrhage. Neurocrit Care 26:48-57, 2017

13. Garcia D, Barrett YC, Ramacciotti E, Weitz JI: Laboratory assessment of the anticoagulant effects of the next generation of oral anticoagulants. J Thromb Haemost 11:245-252, 2013

14. Gonzalez E, Pieracci FM, Moore EE, Kashuk JL: Coagulation abnormalities in the trauma patient: the role of point-ofcare thromboelastography. Semin Thromb Hemost 36:723737,2010

15. Gozal YM, Carroll CP, Krueger BM, Khoury J, Andaluz NO: Point-of-care testing in the acute management of traumatic brain injury: Identifying the coagulopathic patient. Surg Neurol Int 8:48, 2017

16. Haas T, Spielmann N, Mauch J, Madjdpour C, Speer O, Schmugge M, et al: Comparison of thromboelastometry $\left(\right.$ ROTEM $\left.^{\circledR}\right)$ with standard plasmatic coagulation testing in paediatric surgery. Br J Anaesth 108:36-41, 2012

17. Hébert PC, Wells G, Blajchman MA, Marshall J, Martin C, Pagliarello G, et al: A multicenter, randomized, controlled clinical trial of transfusion requirements in critical care. Transfusion Requirements in Critical Care Investigators, Canadian Critical Care Trials Group. N Engl J Med 340:409417, 1999

18. Herrmann R, Thom J, Wood A, Phillips M, Muhammad S, Baker R: Thrombin generation using the calibrated automated thrombinoscope to assess reversibility of dabigatran and rivaroxaban. Thromb Haemost 111:989-995, 2014

19. Hoffman M, Monroe DM III: A cell-based model of hemostasis. Thromb Haemost 85:958-965, 2001

20. Holcomb JB, Minei KM, Scerbo ML, Radwan ZA, Wade CE, Kozar RA, et al: Admission rapid thrombelastography can replace conventional coagulation tests in the emergency department: experience with 1974 consecutive trauma patients. Ann Surg 256:476-486, 2012

21. Hunt H, Stanworth S, Curry N, Woolley T, Cooper C, Ukoumunne $\mathrm{O}$, et al: Thromboelastography (TEG) and rotational thromboelastometry (ROTEM) for trauma induced coagulopathy in adult trauma patients with bleeding. Cochrane Database Syst Rev (2):CD010438, 2015

22. Jansohn E, Bengzon J, Kander T, Schött U: A pilot study on the applicability of thromboelastometry in detecting brain tumour-induced hypercoagulation. Scand J Clin Lab Invest 77:289-294, 2017

23. Johansson PI, Stensballe J, Oliveri R, Wade CE, Ostrowski SR, Holcomb JB: How I treat patients with massive hemorrhage. Blood 124:3052-3058, 2014

24. Kawano-Castillo J, Ward E, Elliott A, Wetzel J, Hassler A, McDonald M, et al: Thrombelastography detects possible coagulation disturbance in patients with intracerebral hemorrhage with hematoma enlargement. Stroke 45:683-688, 2014

25. Kreitzer NP, Bonomo J, Kanter D, Zammit C: Review of thromboelastography in neurocritical care. Neurocrit Care 23:427-433, 2015

26. Kunio NR, Differding JA, Watson KM, Stucke RS, Schreiber MA: Thrombelastography-identified coagulopathy is associated with increased morbidity and mortality after traumatic brain injury. Am J Surg 203:584-588, 2012

27. Laroche M, Kutcher ME, Huang MC, Cohen MJ, Manley GT: Coagulopathy after traumatic brain injury. Neurosurgery 70:1334-1345, 2012

28. Larsen CC, Hansen-Schwartz J, Nielsen JD, Astrup J: Blood coagulation and fibrinolysis after experimental subarachnoid hemorrhage. Acta Neurochir (Wien) 152:1577-1581, 2010

29. Levine J, Kofke A, Cen L, Chen Z, Faerber J, Elliott JP, et al: Red blood cell transfusion is associated with infection and extracerebral complications after subarachnoid hemorrhage. Neurosurgery 66:312-318, 2010

30. Li ZJ, Fu X, Xing D, Zhang HF, Zang JC, Ma XL: Is tranexamic acid effective and safe in spinal surgery? A meta-analysis of randomized controlled trials. Eur Spine J 22:1950-1957, 2013 
31. Lindroos AC, Niiya T, Randell T, Niemi TT: Stroke volumedirected administration of hydroxyethyl starch (HES 130/0.4) and Ringer's acetate in prone position during neurosurgery: a randomized controlled trial. J Anesth 28:189-197, 2014

32. Liotta EM, Garg RK, Temes RE, John S, Lee VH, Bleck $\mathrm{TP}$, et al: Warfarin-associated intracerebral hemorrhage is inadequately treated at community emergency departments. Stroke 43:2503-2505, 2012

33. Luostarinen T, Silvasti-Lundell M, Medeiros T, Romani R, Hernesniemi J, Niemi T: Thromboelastometry during intraoperative transfusion of fresh frozen plasma in pediatric neurosurgery. J Anesth 26:770-774, 2012

34. Massaro AM, Doerfler S, Nawalinski K, Michel B, Driscoll $\mathrm{N}, \mathrm{Ju} \mathrm{C}$, et al: Thromboelastography defines late hypercoagulability after TBI: a pilot study. Neurocrit Care 22:45-51, 2015

35. May AK, Young JS, Butler K, Bassam D, Brady W: Coagulopathy in severe closed head injury: is empiric therapy warranted? Am Surg 63:233-237, 1997

36. McTaggart RA, Choudhri OA, Marcellus ML, Brennan T, Steinberg GK, Dodd RL, et al: Use of thromboelastography to tailor dual-antiplatelet therapy in patients undergoing treatment of intracranial aneurysms with the Pipeline embolization device. J Neurointerv Surg 7:425-430, 2015

37. Mittermayr M, Streif W, Haas T, Fries D, Velik-Salchner C, Klingler A, et al: Hemostatic changes after crystalloid or colloid fluid administration during major orthopedic surgery: the role of fibrinogen administration. Anesth Analg 105:905-917, 2007

38. Moore HB, Moore EE, Liras IN, Gonzalez E, Harvin JA, Holcomb JB, et al: Acute fibrinolysis shutdown after injury occurs frequently and increases mortality: a multicenter evaluation of 2,540 severely injured patients. J Am Coll Surg 222:347-355, 2016

39. Naik BI, Pajewski TN, Bogdonoff DI, Zuo Z, Clark P, Terkawi AS, et al: Rotational thromboelastometry-guided blood product management in major spine surgery. J Neurosurg Spine 23:239-249, 2015

40. Narayan RK, Maas AI, Servadei F, Skolnick BE, Tillinger MN, Marshall LF: Progression of traumatic intracerebral hemorrhage: a prospective observational study. J Neurotrauma 25:629-639, 2008

41. Norton RP, Bianco K, Lafage V, Schwab FJ: Complications and intercenter variability of three-column resection osteotomies for spinal deformity surgery: a retrospective review of 423 patients. Evid Based Spine Care J 4:157-159, 2013

42. Ogawa S, Szlam F, Chen EP, Nishimura T, Kim H, Roback JD, et al: A comparative evaluation of rotation thromboelastometry and standard coagulation tests in hemodilutioninduced coagulation changes after cardiac surgery. Transfusion 52:14-22, 2012

43. Phelan HA: Pharmacologic venous thromboembolism prophylaxis after traumatic brain injury: a critical literature review. J Neurotrauma 29:1821-1828, 2012

44. Qian C, Yan H, Hu X, Zhang W, Liu H: Increased risk of venous thromboembolism in patients with brain tumors: a systematic review and meta-analysis. Thromb Res 137:58-63, 2016

45. Qureshi AI, Luft AR, Sharma M, Guterman LR, Hopkins LN: Prevention and treatment of thromboembolic and ischemic complications associated with endovascular procedures: Part II-Clinical aspects and recommendations. Neurosurgery 46:1360-1376, 2000

46. Raghavan M, Marik PE: Anemia, allogenic blood transfusion, and immunomodulation in the critically ill. Chest 127:295-307, 2005

47. Ramchand P, Nyirjesy S, Frangos S, Doerfler S, Nawalinski $\mathrm{K}$, Quattrone F, et al: Thromboelastography parameter predicts outcome after subarachnoid hemorrhage: an exploratory analysis. World Neurosurg 96:215-221, 2016
48. Reed RL II, Johnson TD, Hudson JD, Fischer RP: The disparity between hypothermic coagulopathy and clotting studies. J Trauma 33:465-470, 1992

49. Robertson CS, Hannay HJ, Yamal JM, Gopinath S, Goodman JC, Tilley BC, et al: Effect of erythropoietin and transfusion threshold on neurological recovery after traumatic brain injury: a randomized clinical trial. JAMA 312:36-47, 2014

50. Rossaint R, Bouillon B, Cerny V, Coats TJ, Duranteau J, Fernández-Mondéjar E, et al: Management of bleeding following major trauma: an updated European guideline. Crit Care 14:R52, 2010

51. Rotondo MF, Cribari C, Smith RS (eds): Resources for the Optimal Care of the Injured Patient. Chicago: American College of Surgeons, 2014 (https://www.facs.org/ /media/ files/quality\%20programs/trauma/vrcresources.ashx) [Accessed September 11, 2017]

52. Salinas D: Viscoelastic studies: effective tools for trauma and surgical resuscitation efforts. AORN J 105:370-383, 2017

53. Salooja N, Perry DJ: Thrombelastography. Blood Coagul Fibrinolysis 12:327-337, 2001

54. Sawaya R, Zuccarello M, Elkalliny M, Nishiyama H: Postoperative venous thromboembolism and brain tumors: Part I. Clinical profile. J Neurooncol 14:119-125, 1992

55. Serraino GF, Murphy GJ: Routine use of viscoelastic blood tests for diagnosis and treatment of coagulopathic bleeding in cardiac surgery: updated systematic review and meta-analysis. Br J Anaesth 118:823-833, 2017

56. Shander A, Hofmann A, Gombotz H, Theusinger OM, Spahn DR: Estimating the cost of blood: past, present, and future directions. Best Pract Res Clin Anaesthesiol 21:271-289, 2007

57. Shen L, Tabaie S, Ivascu N: Viscoelastic testing inside and beyond the operating room. J Thorac Dis 9 (Suppl 4):S299_ S308, 2017

58. Solbeck S, Ostrowski SR, Stensballe J, Johansson PI: Thrombelastography detects dabigatran at therapeutic concentrations in vitro to the same extent as gold-standard tests. Int $\mathbf{J}$ Cardiol 208:14-18, 2016

59. Solomon C, Asmis LM, Spahn DR: Is viscoelastic coagulation monitoring with ROTEM or TEG validated? Scand J Clin Lab Invest 76:503-507, 2016

60. Stein SC, Young GS, Talucci RC, Greenbaum BH, Ross SE: Delayed brain injury after head trauma: significance of coagulopathy. Neurosurgery 30:160-165, 1992

61. Stricker PA, Shaw TL, Desouza DG, Hernandez SV, Bartlett SP, Friedman DF, et al: Blood loss, replacement, and associated morbidity in infants and children undergoing craniofacial surgery. Paediatr Anaesth 20:150-159, 2010

62. Tanaka KA, Bolliger D, Vadlamudi R, Nimmo A: Rotational thromboelastometry (ROTEM)-based coagulation management in cardiac surgery and major trauma. J Cardiothorac Vasc Anesth 26:1083-1093, 2012

63. Velez AM, Friedman WA: Disseminated intravascular coagulation during resection of a meningioma: case report. Neurosurgery 68:E1165-E1169, 2011

64. White N, Bayliss S, Moore D: Systematic review of interventions for minimizing perioperative blood transfusion for surgery for craniosynostosis. J Craniofac Surg 26:26-36, 2015

65. Whiting D, DiNardo JA: TEG and ROTEM: technology and clinical applications. Am J Hematol 89:228-232, 2014

66. Whiting P, Al M, Westwood M, Ramos IC, Ryder S, Armstrong N, et al: Viscoelastic point-of-care testing to assist with the diagnosis, management and monitoring of haemostasis: a systematic review and cost-effectiveness analysis. Health Technol Assess 19:1-228, v-vi, 2015

67. Wikkels $\varnothing$ A, Wetterslev J, Møller AM, Afshari A: Thromboelastography (TEG) or rotational thromboelastometry (ROTEM) to monitor haemostatic treatment in bleeding 
patients: a systematic review with meta-analysis and trial sequential analysis. Anaesthesia 72:519-531, 2017

68. Wikkels $\varnothing$ A, Wetterslev J, Møller AM, Afshari A: Thromboelastography (TEG) or thromboelastometry (ROTEM) to monitor haemostatic treatment versus usual care in adults or children with bleeding. Cochrane Database Syst Rev (8):CD007871, 2016

69. Windel $\varnothing v$ NA, Welling KL, Ostrowski SR, Johansson PI: The prognostic value of thrombelastography in identifying neurosurgical patients with worse prognosis. Blood Coagul Fibrinolysis 22:416-419, 2011

70. Xiao W, Fu W, Wang T, Zhao L: Prophylactic use of tranexamic acid combined with thrombelastogram guided coagulation management may reduce blood loss and allogeneic transfusion in pediatric hemispherectomy: case series. J Clin Anesth 33:149-155, 2016

71. Yang H, Li Y, Jiang Y, Lv X: Thromboelastography for monitoring platelet function in unruptured intracranial aneurysm patients undergoing stent placement. Interv Neuroradiol 21:61-68, 2015

72. Yoshihara H, Yoneoka D: Predictors of allogeneic blood transfusion in spinal fusion in the United States, 2004-2009. Spine (Phila Pa 1976) 39:304-310, 2014
73. Yust-Katz S, Mandel JJ, Wu J, Yuan Y, Webre C, Pawar TA, et al: Venous thromboembolism (VTE) and glioblastoma. J Neurooncol 124:87-94, 2015

\section{Disclosures}

The authors report no conflict of interest concerning the materials or methods used in this study or the findings specified in this paper.

\section{Author Contributions}

Conception and design: Kumar, Schuster. Analysis and interpretation of data: Kumar. Drafting the article: Kumar, Kvint. Critically revising the article: all authors. Reviewed submitted version of manuscript: all authors. Approved the final version of the manuscript on behalf of all authors: Kumar. Study supervision: Kumar.

\section{Correspondence}

Monisha A. Kumar, Department of Neurology, Hospital of the University of Pennsylvania, 3 West Gates Bldg., 3400 Spruce St., Philadelphia, PA 19104.email: monisha.kumar@uphs.upenn.edu. 